\title{
Primary female urethral carcinoma a case report: a review of the diagnosis and treatment
}

\begin{abstract}
Primary urethral carcinoma is a very rare pathology that makes up less than $1 \%$ of all genitourinary tumors. ${ }^{1,2}$ There are several cases report published in the medical literature, although there is no consensus for the management. The prognosis is poor when diagnosed in advanced stages. Treatment includes local or radical surgery with pelvic lymph node dissection, radiotherapy, brachytherapy, and neo or adjuvant chemotherapy. Many authors have suggested a combination of surgery, chemosensibilization and radiotherapy. ${ }^{1,3}$
\end{abstract}

Keywords: urethral carcinoma, diagnosis, tumors

\author{
Volume 2 Issue 3 - 2015
}

\begin{abstract}
Paula Charry G, Ramiro Cabello B, Carlos Simón R, Carmen González Enguita

Urology Department, Hospital Universitario Fundación Jiménez Díaz, Spain
\end{abstract}

\begin{abstract}
Correspondence: Paula Charry G, Urology Department, Hospital Universitario Fundación Jiménez Díaz,Avda Reyes Catolicos 2. 28040 Madrid, Spain, Email pcharry@fjd.es
\end{abstract}

Received: October 21, 2014 | Published: April 09, 20 I I
Abbreviations: UTIs, urinary tract infections; US, ultra sound; MRI, magnetic resonance imaging; TURB, transurethral resection of bladder; CEA, carcino embryonic antigen

\section{Case}

A 54-year old white woman, without significant past medical history other than menopause since 2 years ago presented with post coital hematuria and also she had urinary tract infections (UTIs), documented by urine cultures (Proteus Mirabillis). No other associated symptoms. She received antibiotic therapy with quinolones. The urine cytology was negative. The assessment by the Gynecology service was normal. The renal ultrasound (US) Each kidney was normal, preserved cortico medullary differentiation and without dilated collecting systems. There was irregularity zone in the mucosa close to bladder neck, measuring $1 \times 0.5 \mathrm{~cm}$. Cystoscopy revealed an edematous and erythematosus lesion of $1 \times 1.5 \mathrm{~cm}$ in the posterior wall of the bladder neck that extended into the urethra. In pelvic magnetic resonance imaging (MRI) a pelvic mass was evident measuring $35 \times 38 \times 47 \mathrm{~mm}$, widening the bladder neck at this level, nonspecific inguinal adenopathies and a left iliac node with malignancy aspect.

\section{Initial US}

The pelvic examination during the transurethral resection of bladder (TURB) revealed a palpable nodule on the anterior wall of the vagina and there was not others remarkable findings. The pathology of transurethral resection of bladder confirmed urethra adenocarcinoma muscle-invasive with invasion of bladder neck. She was taken to an extension study (toracoabdominopelvic scan) in which the metastatic lesions were discarded (Figure 1-3).

Radical cystourethrectomy was performed with bilateral pelvic lymph node (extense iliac region) dissection (Figure 4) and anterior vaginal wall excision (vaginoplasty) followed by ileal urinary diversion (Bricker type). Ureteral end edges were sent to frozen biopsy and the results were negative. The specimen's pathology was "adenocarcinoma with clear cell areas and focus of papillary, located in the trigone region, affecting the peri vesical tissues without infiltrating the muscular layer of the vagina (pT4a). Bilateral pelvic lymph nodes were free of tumor (12 nodes in the left side and 14 in the right side), like the rest of organs. Neoplasia stage: pT4a pN0 pM0".

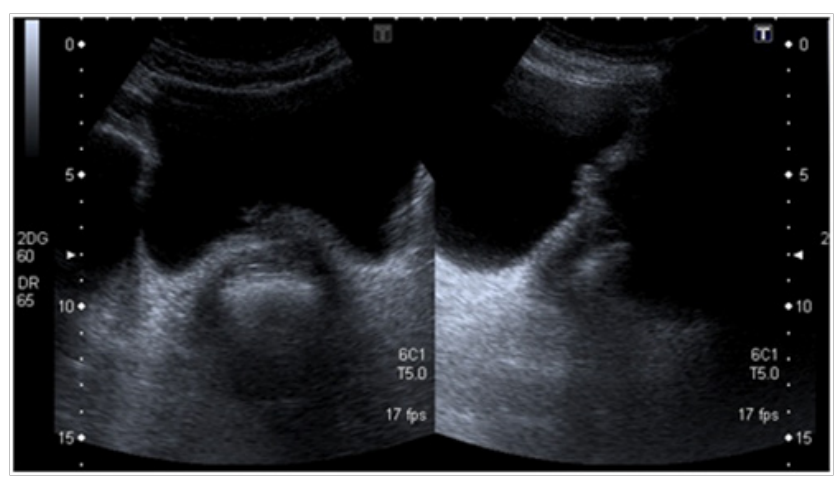

Figure I Initial pelvic ultrasound.

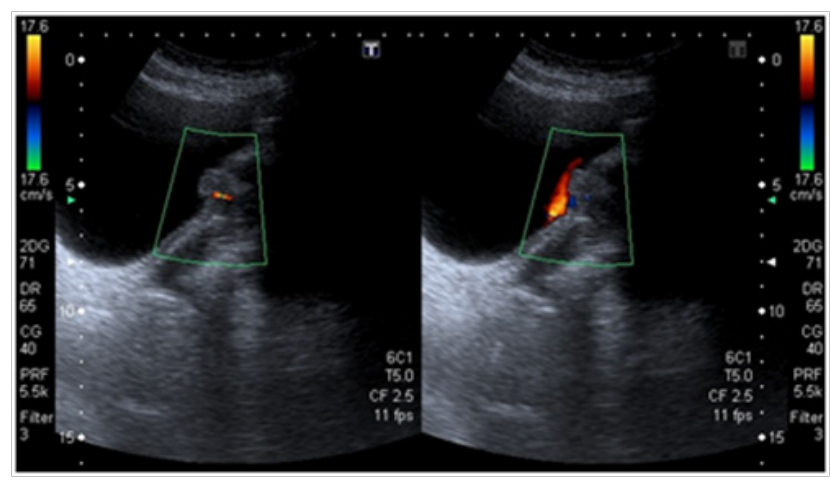

Figure 2 Initial pelvic ultrasound.

After a month postoperative follow- up she was asymptomatic, no findings in the physical examination. So along with Oncologists, she started adjuvant chemotherapy with cisplatine/Taxol/Gemcitabine (3 cycles) during one month. No other complementary treatment. During one year she had oncologic remission with toracoabdominopelvic 
scans and physical examinations every 6months without changes. Twenty months after the surgery in the control scan showed a $42 \times 30 \mathrm{~mm}$ mass in the right pelvic lymphadenectomy area with Ilion infiltration. She also had anemia secondary digestive bleeding. General surgery dismissed curative treatment, for the lesion's localization and the infiltration of piri for muscle (right ciatic nerve compression). She started chemotherapy CDDP/Gem (6 cycles) and right hemi pelvic radiotherapy ( 18 sessions, total doses $4500 \mathrm{cGy}$ ) for pain control. Nowdays, she is finishing the oncology treatment, does not need blood transfusión and also the right leg pain improved.

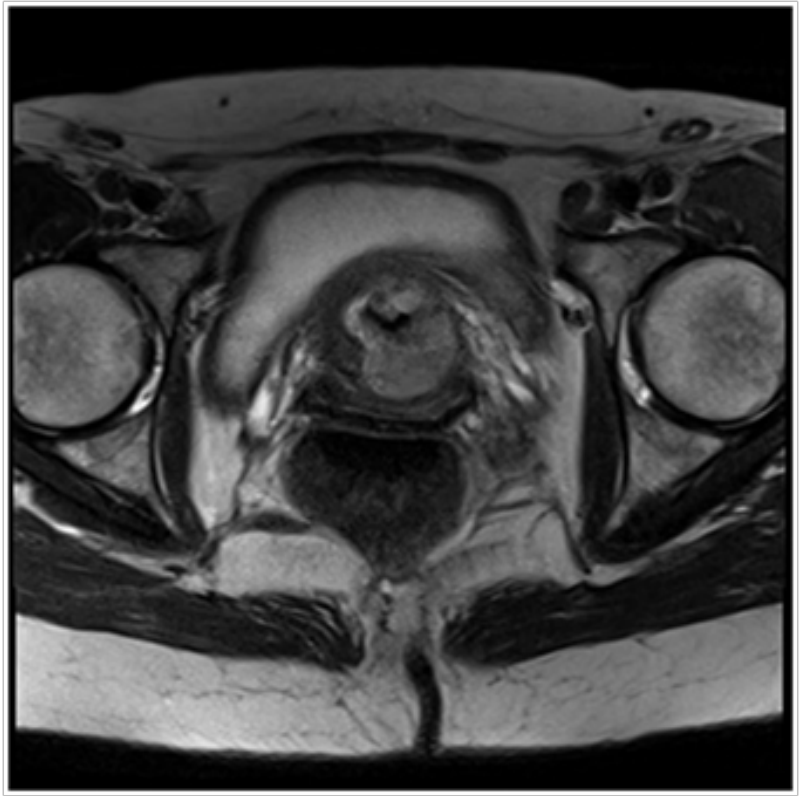

Figure 3 Initial pelvic MRI.

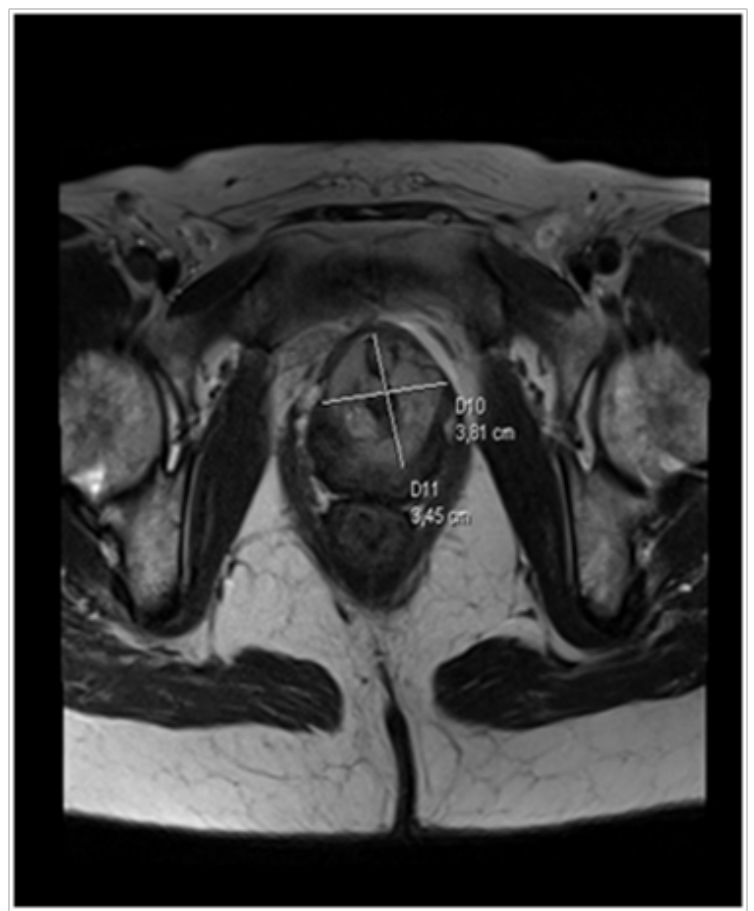

Figure 4 Initial pelvic MRI.

\section{Discussion}

Urethra carcinomas are a very rare pathology (less than $0.02 \%$ in female tumors); most of them are transitional cell carcinoma (55\%), followed by squamous cell carcinoma (21\%) and adenocarcinomas $(16.4 \%){ }^{4}$ The age of onset is over the $50 \mathrm{~s}$ and the most frequent symptoms are bleeding, infections, urethral diverticulum. This pathology is 4 times more common in women compared to men.,

There have been a few reports of urethral carcinomas (Figure 5) diagnosed within dilated paraurethral Skene glands (diverticulo), half of them being adenocarcinomas. ${ }^{7}$ Some authors have proposed that the urethra adenocarcinomas may arise from urethritis glandularis, Mullein ducts (paramesonephric ducts), Skene's glands (paraurethral glands), or mixed origins. The most frequent histological subtype is columnar/mucinous adenocarcinoma and there are around $15 \%$ of clear cell of them. ${ }^{1,3}$

Tumors origins in distal paraurethral duct come from female Skene's glands (homogeneity of male prostate gland) are matched by positive immunostains of PSA and prostatic acid phosphatase. PSA elevation is objective and declines after surgery. The tumors origins in proximal paraurethral ducts can produce carcinoembryonic antigen (CEA). The inmuno histo chemical study can be helpful identifying the type of adenocarcinoma. ${ }^{1,8}$

The diagnosis of this condition can be delayed because of vague and nonspecific symptoms such as hematuria, genital mass feeling, increased urinary frequency, dysuria and urethral obstruction. Urine tract infection with no answer to antibiotic treatment and urine incontinence are less frequently seen as clinical manifestations. ${ }^{1}$ The diagnosis made with urethrocystoscopy and lesion biopsy. The Scan and MRI are made for local and distal extension.

The most important factor for the prognosis/ survival is disease stage, in the early stages the survival in 5years is $50 \%$, in advance disease the survival in 3 years $56 \%$ and there is no survival in 5 years. ${ }^{5,6}$ In the Netherlands, an epidemiological study based on data from the National Cancer Registry during theyears 1989 to 1998, concluded that survival is determined by factors such as the TNM staging and histological type of tumor. The prognosis is poor in advanced stages, when most of them are diagnosed. It has not been possible to establish the most effective treatment for the type of tumor (better prognosis for squamous type and worse if is adenocarcinomas), so the current guidelines are extrapolated from other pathologies. ${ }^{4}$ Other survival factor is the lesion's size, less $2 \mathrm{~cm}$ survival is $60 \%, 2-4 \mathrm{~cm}$ is $46 \%$ and when le lesion is more than $4 \mathrm{~cm}$ the survival is only $13 \%{ }^{5,6}$

The surgical treatment includes a range from local excisions to anterior pelvic exanteration. ${ }^{9,10}$ The local excisions or urethrectomies resulted in postoperative complications, local recurrence or distal metastasis and aggressive treatment as anterior exanteration (specially the posterior urethral lesion), radical cystectomy with urinary diversion and pelvic/groin lymphadenectomy showed promising results with $87 \%$ of recurrence disease- free at 6 months to 2 years. $3,6,11$ In anterior urethral lesions preferred wide extirpation (total or parcialurethrectomy) and if a superficial lesion is, can be treated with local extirpation. ${ }^{5,12}$

For tumors less than $2 \mathrm{~cm}$ curative radiation or surgery with or without adjuvant radiotherapy have been proposed as treatment options, whereas; bulky injury is being treated with brachy (BR) / external beam radiotherapy (RE)(45-50Gy) as a conservative organ 
therapeutic and if there are nodal disease the doses are $60-65 \mathrm{~Gy} ., 5,13$ Moinuddin Ali \& Cols, ${ }^{14}$ treated three patients in early state with RE (40- 50Gy) and extra doses with BR (28-30Gy) presented recurrence disease- free after 30 months. RT as monotherapy could be optimal treatment in early state of distal urethral tumors. ${ }^{10}$ For locally advanced tumors, combined treatment with neo or adjuvant radiotherapy, surgery and chemotherapy, has been associated to cyto reduction. ${ }^{2,4,10}$ There are some series with urethra carcinoma advance state with unfavorable results with surgery or RT as monotherapy. ${ }^{10}$

Dalbagniand Cols, proposed adjuvant RT as part of the surgical treatment with the mean goal to reduce the local recurrence, which improve the disease- specific survival. In the same way, the authors published improvement in the recurrence disease- free after 5year $37 \%$ with neoadjuvant. ${ }^{12}$ A retrospective cohort study has shown that treatment with chemotherapy is beneficial, especially when is given prior to surgery in patients with positive nodes, as it increases disease- free survival. ${ }^{15}$ There are some reports that shown combination therapy with several chemotherapeutic agents in locally advanced female urethral adenocarcinomas like cisplatine- based or cisplatine with 5- fluorouracil or with Carboplatin, plaquitaxel and irinotecane with adjuvant radiotherapy with good results. . $^{1,411,16,17}$

It has proven the Douillard regimen (irinotecane, fluorouracil and leucovorin) in urethral adenocarcinomas and is also used in colon carcinoma and has had evidence in uraco carcinoma as well. ${ }^{1}$ There is a series of 72 women treated at Memorial Sloan-Kettering in a period of 36years, the multivariate analyse showed as independent risk factors for survival the initial stage of tumor, the presence or absence of regional lymph nodes and the site of the disease (proximal versus distal urethra). Being a better prognosis small masses (less than $2 \mathrm{~cm}$ ) in distal urethra, this kind of tumors could be treated with single modality treatment, on the other hand, multimodal treatments has been recommended for larger and proximal tumors. ${ }^{7,18-20}$

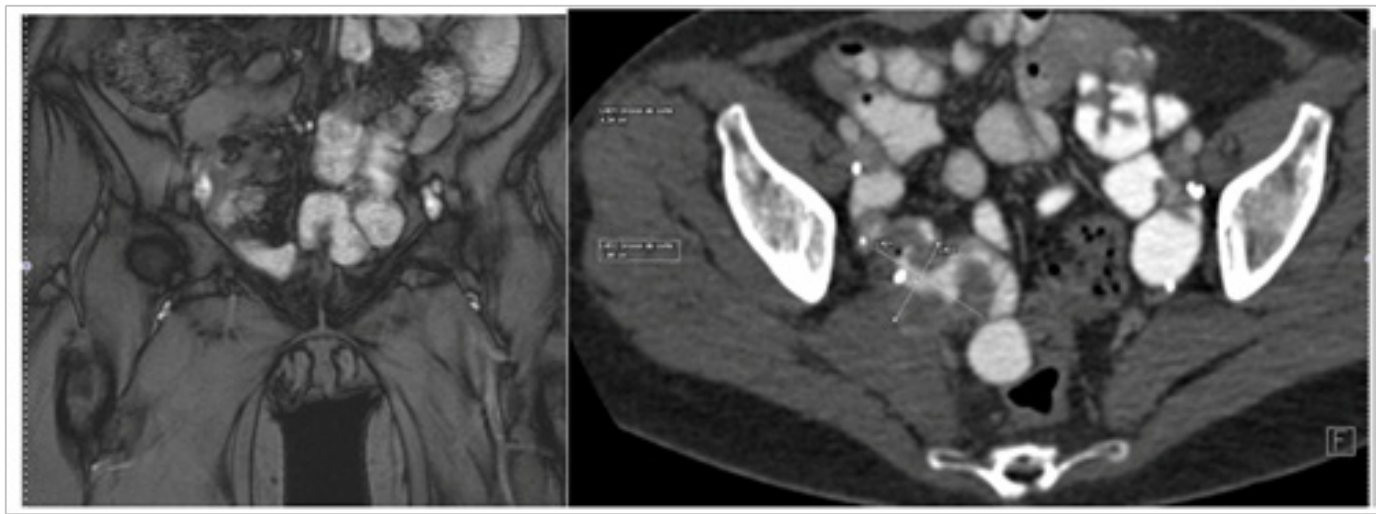

Figure 5 Abdominopelvic CT twenty months after surgery: local recidiva with ilion and right piriform muscle infiltration.

\section{Conclusion}

Does the treatment of urethra carcinoma early states T1 or T2 is surgery with pelvic lymph node dissection and adjuvant RT? Does the treatment of the primary urethral cancer is RT? And the early local recurrence should be removed? Does the chemotherapy is the first line treatment in the primary urethra cancer or should be used with RT or adjuvant treatment in advance state?

The treatment of urethral carcinoma is still controversial, it is a rare condition and there are not enough published retrospective studies, non-prospective studies to clarify. Despite there is not guidelines or protocols for the urethra adenocarcinoma, the combination therapy seems to be the most effective. Besides the histology tumor, the size, location and disease stage are prognostic factors in female urethra carcinoma.

\section{Acknowledgements}

None.

\section{Conflict of interest}

The author declares no conflict of interest.

\section{References}

1. Chen LP, Lin SJ, Fu TY, et al. Locally advanced female urethra adenocarcinoma of entericorigin: The role of adjuvant chemoradiation and brief review. The Kaohsiung J Med Sci. 2011;27(4):150-154.
2. Libby B, Chao D, Schneider BF. Non- Surgical treatment of primary female urethral cancer. Rare Tumors. 2010;2(3):55.

3. Ha HK, Lee W, Lee SD, et al. Laparoscopic Radical Cystourethrectomy in a Patient with Adenocacinoma of Female Urethral Diverticulum. Korean J Urol. 2010;51(2):145-148.

4. Derksen JW, Visser O, De la Rivere GB. Primary Urethral carcinoma in females: An epidemiologic study on demographical factors, histological types, tumour stage and survival. Word J Urol. 2013;31:147-153.

5. García Barreras S, Fiter-Gómez L, Telles-Martínez M. Adenocarcinoma de urethra femenina: presentation de dos casos y revision de la literature. Arch ESP Urol. 2014;67(8):718-721.

6. Miller J, Karnes RJ. Primary clear- cell adenocarcinoma of proximal female urethra: Case report and review of literature. Clin Genitourin Cancer. 2008;6(2):131-133.

7. Salvador Alvarez E, Alvarez Moreno E, Jimenez de la Peña M, et al Malignant degeneration in a urethral diverticulum: an uncommon complication in a common condition. Radiologia. 2011;53(3):266-269.

8. Kawano K, Yano M, Kitahara S, et al. Clear Cell Adenocarcinoma of the female urethra showing strong inmuno staining for prostate-specific antigen. BJU Int. 2001;87(4):412-413.

9. NCCN Guidelines 2014

10. Urologic Clinics of North America. 37(3).

11. Awakura $\mathrm{Y}$, Nonomura $\mathrm{M}$, Itoh $\mathrm{N}$, et al. Adenocarcinoma of female uretral diverticulum treated by multi modality therapy. Int $J$ Urol. 2003;10(5):281-283. 
12. Dalbagni G, Donat SM, Eschwège $P$, et al. Results of high doses rate brachytherapy, anterior pelvic exanteration and external beam radio therapy for carcinoma of the female urethra. J Urol. 2001;166(5):17591761

13. Chan YM, Ka-Leung Cheng D, Nga-Yin Cheung A, et al. Female urethral adenocarcinoma arising from urethritis glandularis. Gynecol Oncol. 2000;79(3):511-514.

14. Moinuddin Ali M, Klein FA, Hazra TA. Primary female urethral carcinoma. A retrospective comparison of different treatment techniques. Cancer. 1988;62(1):54-57.

15. Dayyani F, Pettaway CA, Kamat AM, et al. Retrospective analysis of survival out comes and the role of cisplatine- based chemotherapy in Patients with urethral carcinomas referred to medical oncologists. Urol Oncol. 2013;31(7):1171-1177.
16. Davis R, Peterson AC, Lance R. Clear cell adenocarcinoma in a female urethral diverticulum. Urology. 2003;61(3):644.

17. Dimarco DS, Dimarco CS, Zincke H, et al. Surgical treatment for local control of female urethral carcinoma. Urol Oncol. 2004;22(5):404-409.

18. Nicholson S, Tsang D, Summerton D. Aggressive combined- modality therapy for s squamous cell carcinoma of the female urethra. Nat Clin Pract Urol. 2008;5(10):574-577.

19. Thyavihally YB, Wuntkal R, Bakshi G, et al. Primary carcinoma of female urethra: single center experience of 18 cases. Jnp J Clin Oncol. 2005;35(2):84-87.

20. Milosevic MF, Warde PR, Banerjee D, et al. Urethral carcinoma in women: Results of treatment with primary radio therapy. Radiother Oncol. 2000;56(1):29-35. 